3. Brennan Stephen. Trastornos Renales e hidroelectrolíticos. En Varon J. Cuidados Intensivos. Ed. Mosby/Doyma libros. Madrid 1995; 355-358.

4. Guerola J, Suay AL. Trastornos del Equilibrio Acido-Base. En Manual de Procedimientos de Urgencia y Atención Primaria. Ed. Diputación Provincial de Castellón 1995; 485-491.

5. Kopel RF, Durbin CG Jr. Pulmonary artery catéter deterioration during hydrochlor for the treatment of metabolic alkalosis. Crit Care Med 1989; 17 (7): 688-9.

6. Palmer BF, Alpern RJ. Metabolic alkalosis. J Am Soc Nephrol 1997; 8 (9): 1462-9.

7. Marik PE, Kussman BD, Lipman J, Kraus P. Acetazolamide in the treatmen of metabolic alkalosis in critic patients. Heart Lung 1991; (5 Pt 1): 455-9.

8. Niederstadt C, Kurowski V, Djonlagic H. Severe metabolic alkalosis with a consciousness disorder. Dtsch Med Wochenschr 1996; 121 (3132): $978-82$

\section{Síndrome neuroléptico maligno e hiponatremia relacionados con una intoxicación por risperidona}

\section{Sr. Director:}

El síndrome neuroléptico maligno (SNM) es una complicación seria y potencialmente fatal descrita inicialmente por Delay en los primeros ensayos clínicos realizados con haloperidol (1). La incidencia del SNM se estima entre un 0,5 y un 2,4\% de el total de pacientes tratados con neurolépticos, aunque quizá la incidencia real sea mayor, debido a que muchos de los casos no son adecuadamente diagnosticados (2), Este fallo en el diagnóstico del SNM conduce a que la mortalidad del cuadro sea elevada, llegando a ser de un $20 \%$ (3). La tétrada clásica de presentación del SNM consiste en fiebre, rigidez muscular generalizada, alteraciones del estado mental y disfunción del sistema nervioso autónomo que se manifiestan como hiper o hipotensión, taquicardia, diaforesis e incontinencia urinaria (4). Analíticamente el rasgo más frecuente es la rabdomiolisis que se expresa con elevación de creatinin kinasa en el suero (5). La risperidona es un un neuroléptico que combina el antagonismo hacia los receptores serotoninérgicos y D2 de la dopamina, produciendo menos efectos secundarios, sobre todo de tipo extrapiramidal que otros neurolépticos considerados clásicos (6). Hasta la fecha (búsqueda mediante base de datos MEDLINE 1985-mayo de 1999) tan solo se han descrito 11 casos de síndrome neuroléptico maligno en relación al tratamiento con este fármaco (7). Presentamos el caso de un varón de 34 años de edad, con un trastorno psicótico de larga evolución en tratamiento con risperidona $3 \mathrm{mg}$ al día desde hacía más de 6 meses así como $2 \mathrm{mg}$ al día de loracepam. Diez horas antes de acudir al hospital ingiere con finalidad autolítica $180 \mathrm{mg}$ de risperidona y $15 \mathrm{mg}$ de loracepam. Es trasladado a urgencias, donde llega agitado, con diaforesis franca, no se evidenció rigidez muscular. El paciente estaba febricular (temperatura axilar $37,8^{\circ} \mathrm{C}$ ), taquicárdico a $118 \mathrm{Ipm}$. y con tensión arterial de 110-70 mm Hg. La analítica general mostró 13,2 X109/L leucocitos, con fórmula leucocitaria normal, 5,39X10 12/L hematies, hemoglobina de 18,4 g/dL, hematocrito del 49,8\%, VCM de $92,4,161$ X10 9/L plaquetas. Sodio 133 mmoUL, Potasio 4,2 $\mathrm{mmol} / \mathrm{L}$, glucosa $133 \mathrm{mg} / \mathrm{dL}$, urea $37 \mathrm{mg} / \mathrm{dL}$, creatinina 0,99 $\mathrm{mg} / \mathrm{dL}$, CK $65.950 \mathrm{U} / \mathrm{L}$, con CK-MB de $1185 \mathrm{U} / \mathrm{L}(1,8 \%)$. Gasometria venosa con $\mathrm{pH} 7,44$, bicarbonato $26,7 \mathrm{mmoVL}$, exceso de base de 3,1 $\mathrm{mmol} / \mathrm{L}$. Radiografía de tórax era normal, sedimento de orina sin alteraciones y ECG con un ritmo sinusal a $110 \mathrm{lpm}$, sin alteraciones en el trazado. El enfermo fue tratado con medidas de soporte, hidratacion y carbón activado para disminuir la absorción del tóxico. La clínica de agitación y de disfunción autonómica manifestada por la diaforesis, taquicardia y alteraciones tensionales desapareció en las primeras 24 horas de tratamiento, así como la hiponatremia. Las CK se normalizaron en 48 horas, no presentándose parámetros analíticos que indicasen deterioro de la función renal atribuible a la rabdomiolisis. Consideramos nuestro caso de interés ya que ninguno de los casos de SNM atribuidos a la ingesta de risperidona publicados préviamente eran debidos a ingesta masiva del fármaco. La elevación de las CK en nuestro paciente fue mucho mayor que en el resto de los casos publicados, ya que el nivel máximo de ésta enzima en los casos publicados fué de 19,710 U/L (8). Por otro lado se añade en nuestro paciente la presencia de hiponatremia, que ha sido relacionada con la sobredosificación de risperidona (9).

\section{F. Epelde Gonzalo}

Servicio de Urgencias. Hospital Mutua de Terrassa. Barcelona

1. Delay J, Pichot P, Lemperie T, Elissalde B, Peigne F. Un neuroleptique majeur nonphenothiazine et non- reserpinique, 1 haloperidol, dans le traitement des psychoses. Ann Med Psychol 1960; 118: 145-52.

2. Pope HG, Keck PE, Mc Elroy SL. Frecuency and presentation of neuroleptic malignant syndrome in a large psychriatic hospital. Am J Psychiratry $1986 ; 143: 1227-33$.

3. Freidman JH, Davis R, Wagner R. Neuroleptic malignant syndrome. The result of a 6 month prospective study of incidence in a state psychiatric hospital. Clin Neuropharmacol 1988; 52: 578-86.

4. Crurrera RJ, Chang SS, Romero JA. A comparison of diagnostic criteria for neuroleptic malignant syndrome. J Clin Psychiratry 1992; 53: 56-62.

5. Meltzer HY, Cola PA, Parsa M. Marked elevations of serum creatine kinase activity associated with antipsychotic drug treatment. Neuropsychopharmacology 1996; 15: 395-405.

6. Allison A, Acri Rph, Fred M, Henretig MD. Effects of risperidone in overdose. Am J Emerg Med 1998; 16: 498-501.

7. Sharma R, Trappler B, Kee Y, Leeman C. Risperidone-induced neuroleptic malignant syndrome. Ann Pharmacother 1996; 30: 775-8.

8. Levin GM, Lazowick AL, Powell HS. Neuroleptic malignant syndrome with risperidone. J Clin Psychofarmacol 1996; 16: 192-3.

9. Brown K, Levy H, Brenner C, Leffler S, Hamburg EL. Overdose of risperidone. Ann Emerg Med 1993; 22: 1908-10.

\section{Pericarditis brucelar}

\section{Sr. Director:}

Las complicaciones cardiacas de la brucelosis son infrecuentes, destacando por su mayor gravedad e incidencia la endocarditis. En ausencia de ésta, la pericarditis es excepcional. Presentamos un nuevo caso de pericarditis brucelar (PB).

Varón de 17 años de edad, en contacto con ganado ovino, diagnosticado 4 meses antes de su ingreso de brucelosis. Fue tratado con doxiciclina $(100 \mathrm{mg} / 12 \mathrm{~h})$ y rifampicina $(900 \mathrm{mg} / 24 \mathrm{~h}$.) durante 45 días con buena respuesta clínica. Permaneció asintomático hasta el día de su ingreso, en el que sufrió dolor retroesternal, opresivo, de dos horas de duración. En la exploración física, se encontraba afebril y se auscultó un roce pericárdico. Analítica: CPK 674 UI/l, CPK-MB 50 UI/l, GOT 117 UI/l, GPT 217 UI/l y LDH 587 UI/l. Hemograma: Hb. 14,2 g/dl, leucocitos 5,7 x 10\%/l, plaquetas $195 \times 10^{9} / 1$ Serología de CMV, enterovirus, anticuerpos heterófilos, ANA, crioglobulinas y factor reumatoide negativa. Rx de tórax: normal. ECG: elevación difusa del segmento ST y T (-) de V3-V6. Ecocardiograma: pericardio engrosado, sin derrame pericárdico. Test Rosa de Bengala: positivo; aglutinaciones frente a Brucella, 1/640; Coombs, 1/1280. Hemocultivos (3/3): crecimiento de $B$. mellitensis a los nueve días de incubación. El paciente fue tratado inicialmente con ácido acetil salicílico, y al conocer el diagnóstico etiológico, con rífampicina (900 mg/24 h), 
doxiciclina $(100 \mathrm{mg} / 12 \mathrm{~h})$ y cotrimoxazol $(80-400 \mathrm{mg} / 6 \mathrm{~h})$ que se mantuvo durante 2 meses. La evolución clínica, electrocardíográfica y ecocardiográfica fué favorable, y 12 meses después se encontraba asintomático.

La PB asociada a endocarditis, se describió hace más de 50 años. Sin embargo, los primeros casos de PB aislada no fueron descritos hasta hace dos décadas $(1,2)$. En una revisión realizada en la base de datos Medline, utilizando los términos Pericarditis/Mio carditis y Brucella desde 1982 hasta la actualidad, y por las referencias aportadas por otros estudios, hemos encontrado 10 enfermos con PB (1-8), dos de ellos con participación miocárdica $(6,8)$.

La incidencia de PB es difícil de cuantificar, pero probablemente es excepcional. Así, en una serie de 400 enfermos con brucelosis, se cita un caso de pericarditis (9). En otras publicaciones, no se menciona esta complicación (10). En lo que se refiere a la patogénesis, se han propuesto dos hipótesis: invasión brucelar y depósito de inmunocomplejos $(3,7)$. Es interesante señalar que en los dos únicos pacientes en los que se realizó pericardiocentesis, se obtuvo crecimiento de Brucella sp (2).

Las manifestaciones clínicas varían, desde cuadros leves al taponamiento cardiaco $(2,5,7)$. Son frecuentes el dolor precordial, la fiebre y el roce pericárdico. Los hemocultivos fueron realizados en 10 enfermos (incluyendo el nuestro), creciendo Brucella sp. en 8 de ellos. En ausencia de pericardiocentesis el diagnóstico se basa en la demostración de pericarditis coincidiendo con la existencia de brucelosis, una vez descartadas otras etiologías (3). El tratamiento antibiótico de la brucellosis (tetraciclinas asociadas a estreptomicina o rifampicina), ha proporcionado generalmente buenos resultados, y solo dos pacientes precisaron pericardiocentesis, falleciendo uno de ellos (2).

En resumen, la pericarditis puede ser una de las múltiples manifestaciones de la brucelosis y debe de considerarse, especialmente en regiones donde esta zoonosis es endémica.

\section{A. Chocarro Martínez, A. González López, P. Zuazola, I. García García}

Unidad de Infectología. Servicio de Medicina Interna. Hospital Virgen de la Concha. Zamora

1. Cuisinier Y, Blanc P, Doumeix JJ, Virot P, Chabanier A, Delhoume B, Bensaid J. Péricardite au cours de la brucellose. Nouv Presse Med 1982; 11: 3352-3.

2. Ugartemendia MC, Curós-Abadal A, Pujol-Rakosnik M, Pujadas-Capmany R, Escrivá-Montserrat E, Jané-Pesquer J. Brucella melitensis pericarditis. Am Heart J 1985; 109: 1108.

3. Gómez-Huelgas R, Mora M, Porras JJ, Nuño E, San Román CM. Brucella and acute pericaditis: fortuitous or causal association?. J Infect Dis 1986; 154: 544

4. Rivera JM, García-Bragado F, Gómez FA, Grilo A, Lozano-Gutierrez F. Brucellar pericarditis. Infection 1988; 16: 254.

5. Andrés C, Magallón A, Ochoa FJ, Peña JL, Salcedo FJ, Campo JM. Brucelosis y derrame pericárdico. An Med Interna (Madrid) 1989; 6: 656-7.

6. Delmastro B, Libero L, Bordino C, Birlocchio C, Alfani M, Mantovani M, Casabianca A y D'Anelli A. Su di un caso di pericardite in corso brucellosi acuta. Minerva Med 1989; 80: 1245-9.

7. Anguita M, Díaz V, López-Granados A, Vivancos R, Mesa D, Suárez de Lezo J, Vallès F. Pericarditis brucelósica: dos formas diferentes de presentación para una etiología poco frecuente. Rev Esp Cardiol 1991; 44: 482-4.

8. Marcos F, Vázquez A, Juárez F, López P, Duran A. Afectación pericárdica y miocárdica como forma de presentación de la brucelosis. An Med Interna (Madrid) 1991; 8: 100-1.

9. Lulu AR, Araj GF, Khateeb MI, Mustafa MY, Yusuf AR, Fenech FF. Human brucellosis in Kuwait: a prospective study of 400 cases. Q J Med 1988; 249: 39-54

10. Zancada F, Roldán A, Fernández A, Jiménez FJ, Agulla A. Brucelosis: estudio clínico serológico en un área rural. Rev Clin Esp 1992; 191: 8-12.

\section{Candidiasis diseminada con afectación miocárdica}

\section{Sr. Director:}

Las infecciones cardíacas por hongos son más frecuentes en pacientes que reciben inmunosupresores o antibióticos $(1,2,3)$. En el caso de la candidiasis cardiaca, las principales manifestaciones son la endocarditis y los abcesos miocárdicos múltiples $(2,3)$. La invasión miocárdica por Candida puede complicar sig nificativamente la evolución de la candidiasis sistémica y conducir a la muerte del paciente sin haber llegado a un diagnóstico clínico $(1,3)$. Presentamos un caso de miocarditis por Candida diagnosticado en el estudio de la necropsia.

Varón de 36 años con una neumonía eosinófila crónica dos años antes tratada con corticoides y posteriormente una sinusitis maxilar. En una serología presentó los anti-HBc y los IgG-HC positivos y el VIH negativo. Negaba adicción a drogas por vía parenteral y su hábito enólico era moderado. Ingresó en planta por artromialgias y sensación de acorchamiento en extremidades inferiores de tres semanas de evolución, febrícula, tos y disnea a moderados esfuerzos. A la exploración física estaba afebril y presentaba taquipnea con roncus dispersos, tensión arterial de 120/80 mmHg, ruidos cardiacos sin soplos a $80 \mathrm{lpm}$, hepatomegalia de $2-3 \mathrm{~cm}$, pequeñas costras alrededor de los tobillos y en los codos, fuerza normal y sensibilidad disminuida en las extremidades inferiores. La gasometría con $\mathrm{FiO}_{2} 0,21$ mostró una $\mathrm{pO}_{2} 66 \mathrm{mmHg}$ y en la analítica de sangre destacaba: creatinina 1,2 mg/dl, GOT $80 \mathrm{U} / \mathrm{L}$, GPT $45 \mathrm{U} / \mathrm{L}, \mathrm{LDH} 1137$ $\mathrm{U} / \mathrm{L}, \quad$ K $5.4 \mathrm{mEq} / \mathrm{l}$ y leucocitos $18940 / \mathrm{uL}$ (neut 6500, eosi 9700), siendo el resto normal. En el sedimento de orina tuvo proteinas ++ y 10-15 leucocitos/campo, en la radiografía de tórax un índice cardiotorácico en el límite alto de la normalidad y en el ECG taquicardia sinusal y bloqueo de rama derecha del haz de His (BRDHH). En cinco días desarrolló una insuficiencia respiratoria grave, fiebre e hipotensión arterial de 80/40 $\mathrm{mmHg}$ por lo que ingresó en la Unidad de Cuidados Intensivos, siendo intubado y conectado a ventilación mecánica. En la Rx tórax se observó cardiomegalia e infiltrado alveolar bibasal. Siguió con fiebre, precisando noradrenalina y dobutamina para mantener la tensión arterial. El índice cardíaco siempre fue < $2,21 / \mathrm{min} / \mathrm{m}^{2}$, el ECG mostró complejos de bajo voltaje y un ecocardiograma transtorácico reveló: VId 58 mm, FE 30\%, acinesia de pared posterior e hipocinesia apical. Las CPK/CK-MB seriadas fueron negativas. En un esputo simple se aislaron $>1000000$ UFC $H$. Influenzae iniciando tratamiento antibiótico. Los cultivos de sangre y orina y el de otra muestra bronquial fueron negativos. Al tratamiento con corticoides ya iniciado, se añadió ciclofosfamida pues un estudio de conducción parecía apoyar la sospecha diagnóstica de vasculitis. A los ocho días falleció en situación de shock cardiogénico refractario. La autopsia reveló una candidiasis diseminada con miocarditis aguda extensa (Fig. 1).

Las infecciones cardiacas por hongos están aumentando en número (1) y la Candida es el organismo más frecuente, causando miocarditis o endocarditis (2). La miocarditis por Candida ocasiona microabcesos difusos con elementos levaduriformes y pseudohifas (3 y Fig. 1) resultado de una sepsis diseminada (4). La edad media de estos pacientes es de 37 años (3) y suelen haber recibido antineoplásicos, antibióticos o corticoides, solos o combinados, o han sido sometidos a cirugía abdominal o cardíaca $(1,2,3)$. En el examen post-mortem de 60 pacientes con afectación cardíaca por hongos (2) el grupo sometido a tratamiento inmunosupresor o antibiótico tuvo una mayor incidencia de miocarditis (79\%) frente a los quirúrgicos que presentaban una mayor incidencia de endocarditis $(93 \%)$. La invasión cardíaca por Candida puede crear alteraciones de la conducción, arritmias, insuficiencia cardíaca con hipotensión o shock y muerte del paciente $(3,4)$. En una serie de 51 pacientes (1) dos casos con miocarditis candidiásica extensa 